\title{
Scaling and super-cooling in heat storage harvesting devices
}

\author{
M. E. Kiziroglou*a,d, A. Elefsiniotis ${ }^{\mathrm{b}}$, N. Kokorakis ${ }^{\mathrm{b}}$, S. W. Wright ${ }^{\mathrm{a}}$, T. T. Toh ${ }^{\mathrm{a}}$, P. D. Mitcheson ${ }^{\mathrm{a}}$, \\ U. Schmid ${ }^{\mathrm{c}}$, Th. Becker ${ }^{\mathrm{b}}$ and E. M. Yeatman ${ }^{\mathrm{a}}$
}

\begin{abstract}
Aircraft sensors are typically cable powered, imposing a significant weight overhead. The exploitation of temperature variations during flight by a phase change material (PCM) based heat storage thermoelectric energy harvester, as an alternative power source in aeronautical applications, has recently been flight tested. In this work, the applicability of this technology to use cases with smaller and larger size specifications is studied by fabrication, testing and analysis of a scaled-down and a scaled-up prototype. Output energy of 4.1 J per gram of PCM from a typical flight cycle is demonstrated for the scaled-down device, and 2.3 J per gram of PCM for the scaled-up device. The higher energy density of the scaled down prototypes is attributed to the reduction in temperature inhomogeneity inside the PCM. The impact of supercooling on performance is analyzed by employing a simulation model extended to include super-cooling effects. It is found that super-cooling may be beneficial for scaling down, in applications with slow temperature fluctuations.
\end{abstract}

Keywords: energy harvesting, thermoelectric, aircraft, sensors, phase change materials, heat storage

e-mail: M. E. Kiziroglou

m.kiziroglou@imperial.ac.uk

aDepartment of Electrical and Electronic Engineering, Imperial College London, SW7 2AZ, U.K.

${ }^{\mathrm{b}}$ Airbus Group Innovations, Communication and Sensor Department, 81663, Munich, Germany

'Institute of Sensor and Actuator Systems, Vienna University of Technology, 1040 Wien, Austria

${ }^{\mathrm{d}}$ Department of Automation Engineering, Alexander Technological Educational Institute of Thessaloniki, 57400, Greece

\section{Introduction}

Energy harvesting as the technology of collecting environmental energy that is locally available to power microsystems such as sensors was proposed at the beginning of the 2000s (Glynne-Jones et al. 2004; Mitcheson et al. 2004; Shenck and Paradiso 2001; Sodano et al. 2005) . Fifteen years on, a wealth of different approaches and device implementations have been proposed for exploiting motion, heat gradients, RF radiation and light sources. The exploitation of such approaches in useful applications requires that the net available power density is high enough, and that it is available at the desired location, for the desired period of time. In thermoelectric energy harvesting, this translates to the requirement for a temperature difference $\Delta \mathrm{T}$ at the location of installation. Temperature differences are prevalent in industrial environments or in operating engines but they do not necessarily always occur at the required location. This has been a critical limitation in the application of thermoelectric harvesting devices.

For the case of environments where the temperature fluctuates considerably with time, a dynamic thermoelectric harvesting approach has been proposed, using a heat storage unit containing a phase change material (PCM) to induce temperature hysteresis, thereby creating an artificially increased $\Delta \mathrm{T}$ internal to the device compared to an approach applying a sensible heat storage material. Based on this approach, various prototypes have been reported and the operation and performance of such devices has been analysed (Kiziroglou et al. 2014). For aircraft monitoring applications, where a considerable temperature cycle occurs during flight, tests have been performed for the characterisation of power generation performance (Elefsiniotis et al. 2013), and another flight test is planned for the demonstration of energy harvester-powered wireless strain monitoring (Toh et al. 2014). The use of dynamic thermoelectric harvesting has also been considered in countryside environments for applications such as precision agriculture (Papachristou et al. 2013). 
The typical volume of the PCM used in prototypes reported so far is in a range of around $20 \mathrm{~cm}^{3}$. In order to assess the applicability of such devices to different sensor types or application scenarios, it would be useful to know how the performance of these devices scales with size. Therefore, in this work, two new heat storage thermoelectric harvesting devices are presented: one smaller and one larger than the typical size of devices already reported in the literature. The change of PCM volume is approximately an order of magnitude for both. In this way, a first assessment of scalability of this device concept is obtained.

In the following sections, the concept of dynamic thermoelectric harvesting is briefly summarized and then the scaled down and the scaled up prototypes are presented. The results are analyzed by comparison with simulation results obtained using a previously developed numerical model, extended here to account for super-cooling effects. The results are discussed and compared with previously reported devices. Finally, a functional demonstrator, a microcontroller operating while directly powered from the heat storage harvester using a voltage booster and regulator is presented, and conclusions are drawn about the potential use of dynamic thermoelectric harvesting in commercial applications.

\section{Device Concept}

The principle of operation of heat storage thermoelectric harvesting devices has been discussed in detail in previous publications (Becker et al. 2014; Kiziroglou et al. 2014) .

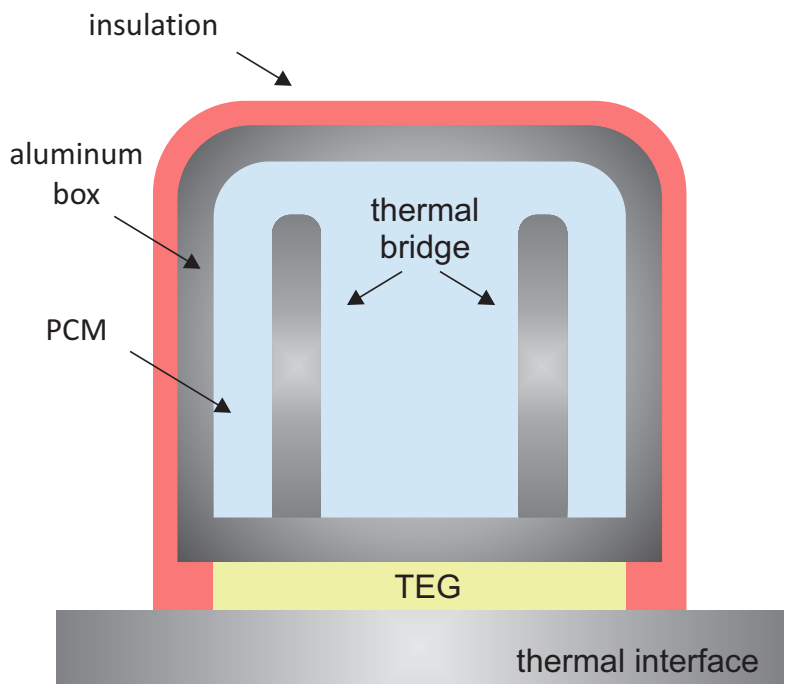

Fig. 1. Internal structure of phase change thermal harvesters
For easy reference, the concept is summarized here, with reference to Figure 1. The device objective is to transform thermal energy from the temperature fluctuation of an environment into electrical energy. A high heat capacity heat storage unit (HSU) is employed, containing a PCM that changes phase within the operating temperature range, thereby increasing the induced heat flow. The HSU is in thermal contact with the environment only through a thermoelectric generator (TEG). As the environmental temperature fluctuates, heat flows in and out of the HSU through the TEG, resulting in generation of electrical power. The high heat capacity of the HSU and, critically, the phase change (i.e. latent) heat of the PCM ensures that a considerable $\Delta \mathrm{T}$ is built up across the TEG.

The temperature and phase change uniformity inside the HSU can be enhanced by metal thermal bridging, such that any $\Delta \mathrm{T}$ loss across the PCM is minimized. The overall power output of such devices scales with $\Delta \mathrm{T}^{2}$, because both the heat flow and the TEG efficiency scale approximately linearly with $\Delta \mathrm{T}$. In voltage terms, this is equivalent to saying that the voltage output of a TEG is proportional to $\Delta \mathrm{T}$ and hence output power scales with $\Delta \mathrm{T}^{2}$. An analysis of the dynamics of operation and performance of this type of device has been presented in (Kiziroglou et al. 2014) and (Kiziroglou et al. 2013) .

\section{Efficiency for maximum power}

A summary of efficiency considerations that are important particularly for heat storage energy harvesting devices is presented in this section. If $\mathrm{T}_{\mathrm{h}}, \mathrm{T}_{\mathrm{c}}$ and $\mathrm{T}=\left(\mathrm{T}_{\mathrm{h}}+\mathrm{T}_{\mathrm{c}}\right) / 2$ are respectively the hot side, cold side and average TEG temperatures, the efficiency as a function of the ratio $\mu$ of load resistance $R_{L}$ over the internal electrical resistance of the TEG, $\mathrm{R}_{\mathrm{e}}$ can be written as:

$$
\eta_{T E G}=\frac{\Delta T}{T_{h}} \cdot \frac{\mu}{\frac{(1+\mu)^{2}}{Z T_{h}}+(1+\mu)-\frac{\Delta T}{2 T_{h}}}
$$

The maximum efficiency occurs when $\mathrm{R}_{\mathrm{L}}$ is such that $\mu=\sqrt{1+Z T}$, giving:

$$
\eta_{T E G, \max }=\frac{\Delta T}{T_{h}} \cdot \frac{\sqrt{1+Z T}-1}{\sqrt{1+Z T}+\frac{T_{c}}{T_{h}}}
$$

where $Z T=a^{2} T /\left(R_{e} K\right)$ is the TEG figure of merit, a its Seebeck coefficient and $\mathrm{K}$ its heat conductance. This operation point is different from the maximum output power point, which occurs for $\mu=1$, giving:

$$
\eta_{T E G, P \max }=\frac{\Delta T}{T_{h}} \cdot \frac{1}{2+\frac{4}{Z T_{h}}-\frac{\Delta T}{2 T_{h}}}
$$


The difference originates from the influence of the Seebeck and ohmic effects on the heat flow, represented by the second and third terms in the denominator of equation (1). These effects result in lower heat flow for a given $\Delta \mathrm{T}$. In other words, the return of the electrical power back to heat through these effects, results in an increase of the actual thermal resistance of a TEG and in a difference between the points of maximum conversion efficiency and maximum power delivery. In conventional TEG applications, the output power is often more important. In contrast, in heat storage thermoelectric harvesting efficiency plays a more significant role, because the total available heat is limited. The optimal electrical load for maximum cumulative energy therefore depends on the heat storage dynamics, especially if the transduction efficiency is high enough for a significant heat flow modulation. A comprehensive analysis of this effect would need to include the heat leakage of the HSU. Overall, this shift of the optimal operation point may allow an increase of performance and should be taken into account in the design and implementation of heat-storage based power supplies. A more detailed analysis of thermoelectric conversion efficiency can be found in chapters 4,6 and 20 of (Briand et al. 2015).

In the evaluation of the devices that are presented in this paper, the maximum power delivery operation point was used, because for the relevant $\mathrm{ZT}$ values and $\Delta \mathrm{T}$ range, the corresponding efficiency difference is less than $6 \%$, and the gain in cumulative energy output is expected to be reduced by heat leakage from the HSU to the environment.

\section{Numerical modeling including super cooling}

A numerical model for heat storage thermoelectric harvesting devices has been introduced in (Kiziroglou et al. 2014), and extended to include temperature inhomogeneity effects in the PCM in (Kiziroglou et al. 2013). According to this model, which includes temperature inhomogeneity in the PCM, a numerical simulation of the system dynamics is possible by calculating every new state $\left(T_{i n}(n+1)\right.$, $\dot{Q}(n+1))$ from the previous $\left(T_{i n}(n), \dot{Q}(n)\right)$ and a given $\mathrm{T}_{\text {out }}$ profile, for a time step $\Delta \mathrm{t}$, using the following equations:

$$
\begin{gathered}
T_{\text {in }}(n+1)=\left\{\begin{array}{c}
T_{\text {in }}(n)+\left(T_{\text {out }}(n)-T_{\text {in }}(n)\right) \cdot \frac{\Delta t}{R C} \text { (non phase change) } \\
T_{\text {in }}(n)+\frac{\left(T_{\text {out }}-T_{\text {in }}\right)^{2}}{R^{2} k \rho L A^{2}} \Delta t \quad \text { (phase change) }
\end{array}\right. \\
\dot{Q}(n+1)=\dot{Q}(n)+\left(T_{\text {out }}(n)-T_{\text {in }}(n)\right) \cdot \frac{\Delta t}{R C}
\end{gathered}
$$

In these equations $\mathrm{R}$ and $\mathrm{C}$ are the thermal resistance between the HSU and the environment, and the thermal capacity of the HSU respectively. The parameters $k, \rho$ and $\mathrm{L}$ are the heat conductivity, density and latent heat of the
PCM respectively. The parameter A is the surface area of the TEG heat sink in the HSU. The electrical power and cumulative energy output can then be calculated by:

$$
\begin{aligned}
& P(n)=\eta_{T E G}(n) \cdot \dot{Q}(n) \\
& E(n)=\sum_{n=1}^{n=\max } P(n) \cdot \Delta t
\end{aligned}
$$

The efficiency $\eta_{\text {TEG }}$ can be calculated from (2), if the ZT of the TEG used in known. A more direct way to calculate the electrical power is through the Seebeck coefficient of the device. If $\mathrm{a}$ and $\mathrm{R}_{\mathrm{i}}$ are known, then the maximum output power will be provided on a matched load $\mathrm{R}_{\mathrm{L}}=\mathrm{R}_{\mathrm{e}}$ (i.e. $\left.\mu=1\right)$, at a value of:

$$
P=\frac{V^{2}}{4 R_{e}}=\frac{a^{2} \Delta T^{2}}{4 R_{e}}
$$

and, for the numerical model:

$$
P(n)=\frac{a^{2}}{4 R_{e}}\left(T_{\text {out }}(n)-T_{\text {in }}(n)\right)^{2}
$$

In practise, the latter method can be more accurate, because the effective Seebeck coefficient of a particular device can be determined directly by a linear fit of experimental $\mathrm{V}-\Delta \mathrm{T}$ measurements. This method will be used in the analysis of the experimental results presented in sections 5 and 6.

In previous implementations, the switching between phase change and non-phase-change operation was done by comparing $\mathrm{T}_{\text {in }}$ to the phase change temperature $\mathrm{T}_{\mathrm{PC}}$. When $\mathrm{T}_{\mathrm{PC}}$ is reached, the model enters its phase change mode, until the PCM latent heat is exhausted. For the work presented here, an extension of this model was developed, to account for super-cooling effects. This extension allows the specification of a super-cooling temperature level $\mathrm{T}_{\mathrm{SC}}$, which needs to be reached before phase change occurs. This additional level applies only during a cooling-down stage. When the super-cooling level is reached, phase change is triggered and the PCM changes abruptly its temperature to the phase change temperature. Then, the non-homogeneous phase change begins, but with a smaller-than-normal remaining latent heat. This difference is equal to the amount of sensible heat that was released during super-cooling. Physically, this reflects the effect that the transition from a super-cooling condition to the phase change temperature $\mathrm{T}_{\mathrm{PC}}$ is accompanied by energy transfer from a latent to a sensible form, such that the liquid can increase its temperature to $\mathrm{T}_{\mathrm{PC}}$. The amount of this energy is $\left(\mathrm{T}_{\mathrm{PC}}-\right.$ $\left.\mathrm{T}_{\mathrm{SC}}\right) \cdot \mathrm{C}$, where $\mathrm{C}$ is the heat capacity of the PCM in liquid form. 


\section{Scaled down prototype}

The size of previously reported heat storage harvesting prototypes is around $100 \mathrm{~cm}^{3}$, with a PCM material volume in the range of $10-30 \mathrm{~cm}^{3}$. In this section, a scaled-down prototype is presented, based on two different TEG models. The first is the Marlow NL1013T $13 \times 13 \times 2.4 \mathrm{~mm}$ TEG, with an internal electrical resistance of $7.42 \Omega$ and a thermal resistance of $16.9 \mathrm{~K} / \mathrm{W}$. This corresponds to a thermal conductivity of $0.68 \mathrm{~W} / \mathrm{m} \cdot \mathrm{K}$. The second is the Eureca TEG1-9.1-9.9-0.8/200 with size $9.1 \times 9.9 \times 2.3 \mathrm{~mm}$, internal electrical resistance $8.85 \Omega$ and thermal resistance $30 \mathrm{~K} / \mathrm{W}$. This corresponds to a thermal conductivity of $0.85 \mathrm{~W} / \mathrm{m} \cdot \mathrm{K}$. The specifications of the TEGs used are summarised in Table I.

The heat storage unit comprises an extruded polystyrene (XPS) lid-less box with outer dimensions $19 \times 19 \times 27 \mathrm{~mm}$ and inner dimensions $10 \times 10 \times 19 \mathrm{~mm}$ and a 9-fin $14 \times 14$ $\times 20 \mathrm{~mm}$ aluminum thermal bridge. The total HSU capacity, taking into account the thermal bridge displacement, is $1.8 \mathrm{ml}$. For the experiments, $1.4 \mathrm{ml}$ of water was used as the PCM. A photograph of the device, with the Marlow TEG installed, is shown in Fig. 2 (left). The thermal bridge is protruding from the box for illustrative purposes. In Fig. 2 (right), a photograph of the device under characterization is shown, featuring the insulated TEG and Pt100 sensor connectors.

The device was characterized in an environmental chamber for various different temperature cycles and with the aircraft environment as an indicative application case. The temperature profiles used were based on previously reported data for an aircraft fuselage during flight (Elefsiniotis et al. 2013). The TEG output voltage was monitored across a connected $7.5 \Omega$ matched load. It is noted here that, as has been demonstrated in (Kiziroglou et al. 2014) and summarized in section 3, the optimal load for maximum power is not the same as for maximum efficiency. In the experiments presented in this paper, the devices were tested under maximum power (i.e. matched load) conditions, for simplicity.

The temperature response of the scaled-down prototype with the Marlow TEG installed is presented in Figure 3. The environmental and the HSU temperature are denoted as $\mathrm{T}_{\text {out }}$ and $\mathrm{T}_{\text {in }}$ respectively. The Tout cycle was from $+22{ }^{\circ} \mathrm{C}$ to $-25{ }^{\circ} \mathrm{C}$ and back, with a temperature change rate of approximately $3{ }^{\circ} \mathrm{C} / \mathrm{min}$, similar to that observed during typical flight scenarios.

Table I. Summary of specifications for the TEGs used in the two scaled-down and the scaled-up prototypes.

\begin{tabular}{llcccc}
\hline Manufacturer & Model & Dimensions [mm] & $\begin{array}{c}\text { Internal electrical } \\
\text { resistance }\end{array}$ & $\begin{array}{c}\text { Thermal } \\
\text { resistance }\end{array}$ & $\begin{array}{c}\text { Thermal } \\
\text { conductivity }\end{array}$ \\
\hline Marlow & NL1013T & $13.2 \times 13.2 \times 2.4$ & $7.42 \Omega$ & $16.4 \mathrm{~K} / \mathrm{W}$ & $0.85 \mathrm{~W} /(\mathrm{m} \cdot \mathrm{K})$ \\
\hline Eureca & TEG1-9.1-9.9-0.8/200 & $9.1 \times 9.9 \times 2.3$ & $8.85 \Omega$ & $30 \mathrm{~K} / \mathrm{W}$ & $0.85 \mathrm{~W} /(\mathrm{m} \cdot \mathrm{K})$ \\
\hline Marlow & TG12-6L & $40.1 \times 40.1 \times 3.9$ & $3.8 \Omega$ & $1.58 \mathrm{~K} / \mathrm{W}$ & $1.54 \mathrm{~W} /(\mathrm{m} \cdot \mathrm{K})$ \\
\hline
\end{tabular}
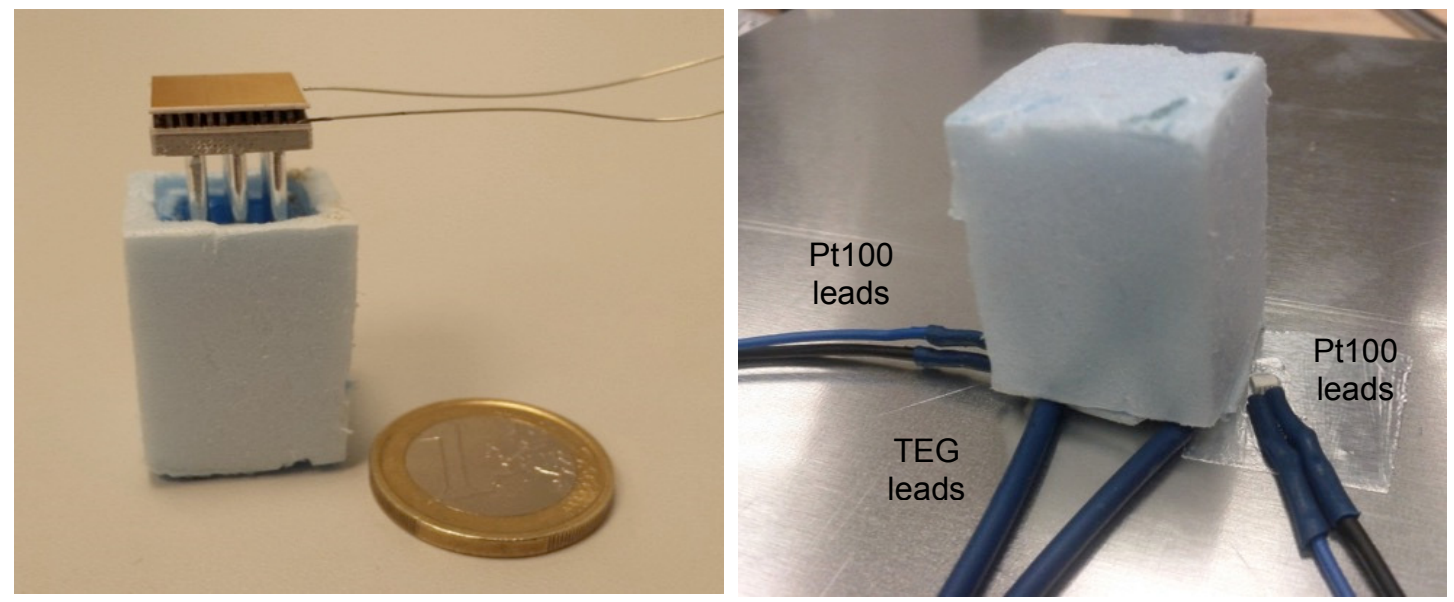

Fig. 2. Images of the scaled down prototype. Left: open device exhibiting the extruded polystyrene (XPS) heat storage unit, the internal heat sink and the TEG. Right: Device under test. 


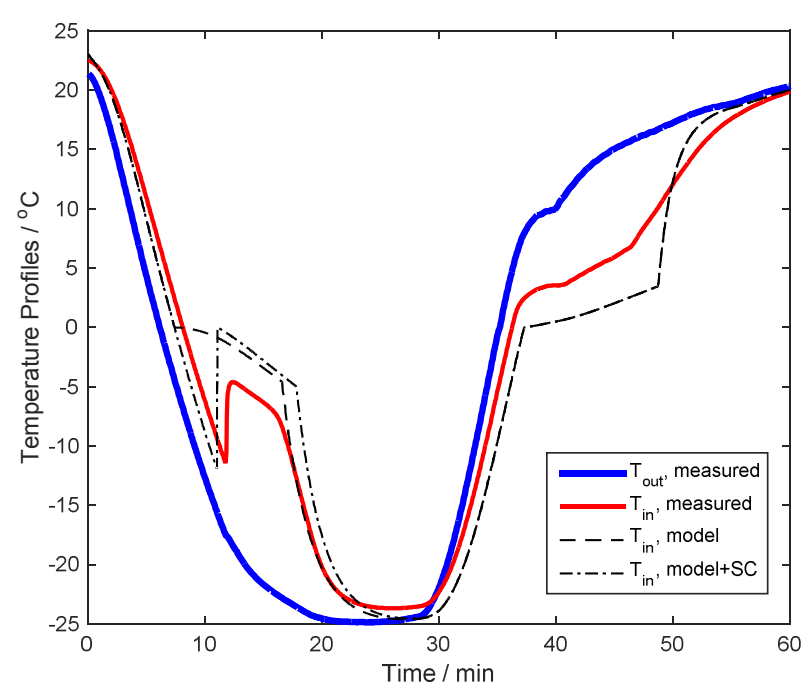

Fig. 3. Temperature response of the scaled-down prototype $(1.4 \mathrm{ml}$ of PCM) during a typical flight temperature cycle using the Marlow TEG. The environmental and HSU temperatures are denoted as $\mathrm{T}_{\text {out }}$ and $\mathrm{T}_{\text {in }}$ respectively. Corresponding model responses using the inhomogeneous phase change model with and without super-cooling (SC) are also shown.

A $\Delta \mathrm{T}$ as high as $15^{\circ} \mathrm{C}$ is achieved during the cooling down phase, with significant water super-cooling. During the warm up phase, the $\Delta \mathrm{T}$ is substantially smaller, mainly due to the slower change of temperature that can be achieved by the environmental chamber. This is due to the large heat absorption from the HSU that occurs during phase change. The large temperature difference that is created results in a high heat flux which disrupts the temperature uniformity in the environmental chamber. In turn, this delays the response of the temperature control system, leading to a slower and distorted $\mathrm{T}_{\text {out }}$ profile during warm-up that is observed between minutes 35 and 45 in Figure 3. Such effects do not occur in a flight environment, because there, the heat sink of the HSU is the fuselage of the aircraft, with a practically unlimited heat capacity. This means that in an applied environment, and provided that a good thermal contact is achieved by the installation method used, the $\mathrm{T}_{\text {out }}$ profile experienced by the harvesting device is not affected by the operation of the device itself.

The temperature response of the device was also simulated, using the model presented in section 3. The results are shown as a dashed curve in Figure 3, using asmeasured parameter values. In particular, for the HSU thermal resistance $\mathrm{R}$, the nominal value of the TEG thermal resistance $(16.4 \mathrm{~K} / \mathrm{W})$ was used. Heat leakage through the polystyrene insulation was not taken into account. For the HSU heat capacity $\mathrm{C}$, both the sensible heat of the PCM and that of the aluminium heat bridge were taken into account. For water, a specific heat capacity of $4.2 \mathrm{~kJ} / \mathrm{kg} \cdot \mathrm{K}$ in liquid form and $2 \mathrm{~kJ} / \mathrm{kg} \cdot \mathrm{K}$ in solid form was assumed, and a latent heat of $\mathrm{L}=334 \mathrm{~kJ} / \mathrm{kg}$ (Kiziroglou et al. 2013). For aluminium, a heat capacity of $0.9 \mathrm{~kJ} / \mathrm{kg} \cdot \mathrm{K}$ was assumed. The device response to the experimentally measured $\mathrm{T}_{\text {out }}$ cycle can then be simulated, accounting for the $1.4 \mathrm{~g}$ of water, the aluminium heat bridge mass which was measured to be $1.72 \mathrm{~g}$, the PCM-Al interface surface of $684 \mathrm{~mm}^{2}$ and taking the nominal heat conductivity value for water, $0.58 \mathrm{~W} / \mathrm{m} \cdot \mathrm{K}$ as the effective conductivity during phase change. Parametric fitting was avoided to maintain simplicity in the interpretation of the comparison.

The simulation curve follows the different effects that are captured by the measured data, and shows good matching in the exponential cooling down stage gradient, in the inhomogeneous phase change temperature gradient and the phase change duration. The simulation model used for the dashed curve on Figure 3 does not include supercooling. The introduction of super-cooling to the model is possible. Such a simulation is shown as a dash-dotted line in Figure 3. This effect and its importance to the performance of heat storage thermoelectric harvesting device will be discussed in section 5 .

The corresponding TEG output voltage, measured using a data logging multimeter across a $7.5 \Omega$ load, and the corresponding cumulative energy, calculated directly as $E=\int V^{2} / R_{L} d t$ are presented in Figure 4. The total cumulative energy harvested from a full temperature cycle was $5.8 \mathrm{~J}$. This corresponds to an energy density of $4.14 \mathrm{~J}$ per gram of PCM, around $10 \%$ lower than the corresponding $4.57 \mathrm{~J} / \mathrm{g}$ that has been previously reported for a device with $23 \mathrm{~g}$ of PCM. The electrical power and energy output that correspond to the simulated device response can

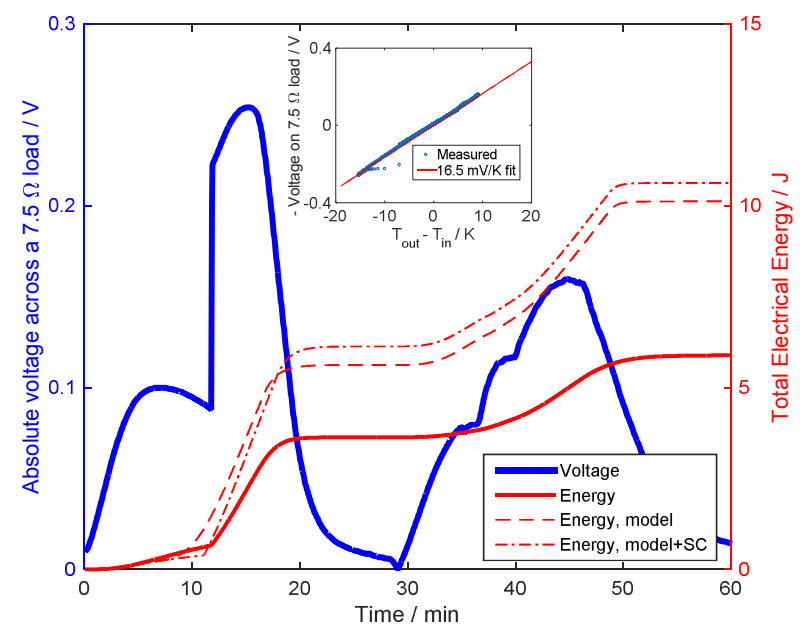

Fig. 4. Voltage and energy output, on a $7.5 \Omega$ load, of the scaled down prototype corresponding to the temperature cycle of Figure 3. The corresponding modeled energy performance is also plotted, using an effective Seebeck coefficient of $16.5 \mathrm{mV} / \mathrm{K}$ which was determined from fitting the $\mathrm{V}-\Delta \mathrm{T}$ measurements as shown in the inset. 
be calculated either from the heat power flow, using the TEG efficiency equation at optimum load operation, or through the determination of an effective Seebeck coefficient for the HSU-TEG system, which can be found by a linear fit of the measured voltage $-\Delta \mathrm{T}$ data.

Such a fit for the device under study is shown in the inset of Figure 4. A TEG output of $16.5 \mathrm{mV} / \mathrm{K}$ is found across a $7.5 \Omega$ load. As this load value matches the internal resistance of the TEG, the corresponding open-circuit voltage is twice the closed-circuit one. Hence, the effective Seebeck coefficient of the device is $33 \mathrm{mV} / \mathrm{K}$. The corresponding simulated energy curves, for the model without and with super-cooling, are shown as a dashed and a dash-dot curve in Figure 4 respectively. These curves both predict a higher output energy for this device. This deviation can be attributed to temperature inhomogeneity which creates a lag between the temperature in the PCM bulk and the temperature at the sensor location, which shows phase change at around $-5^{\circ} \mathrm{C}$ at cool-down, and around $+2{ }^{\circ} \mathrm{C}$ during warm up. This error in the temperature measurements leads to an over-estimation of the effective Seebeck coefficient from the $\mathrm{V}-\Delta \mathrm{T}$ fit, and in turn, to the cumulative energy being over-estimated.

Similar results are obtained using the Eureca TEG, connected to a matched $8.8 \Omega$ load. The temperature response of this device to a flight temperature cycle is shown as a light (red in the online version) curve in Fig. 5. The experimental results are again compared with a simulation of device performance, using a numerical model which includes a non-homogeneous phase change approximation, with and without super cooling. All parameters were the same as with the simulation run of

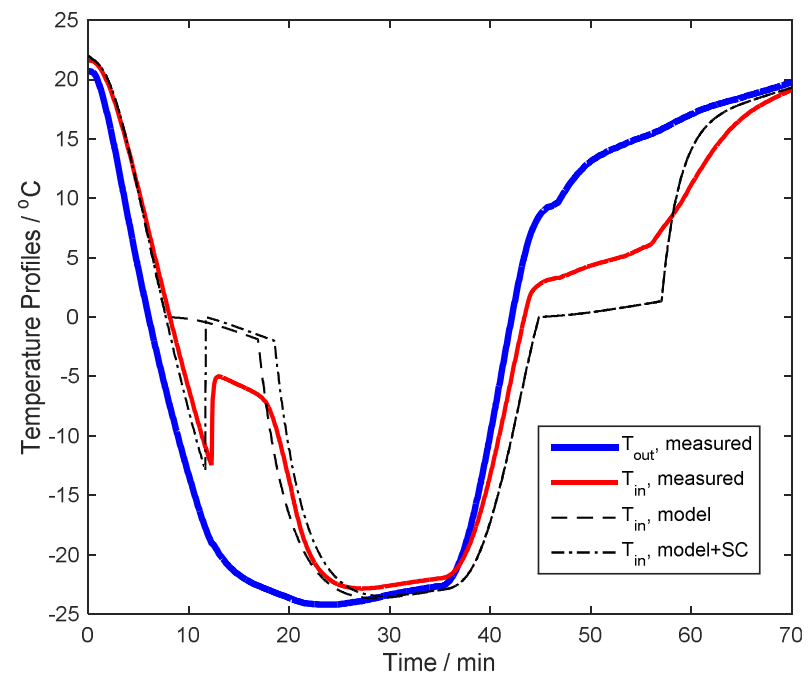

Fig. 5. Temperature response of the scaled-down prototype during a typical flight temperature cycle using the Eureca TEG. The corresponding model responses are also shown for comparison, with a fitted PCM volume value of $0.9 \pm 0.1 \mathrm{~cm}^{3}$.

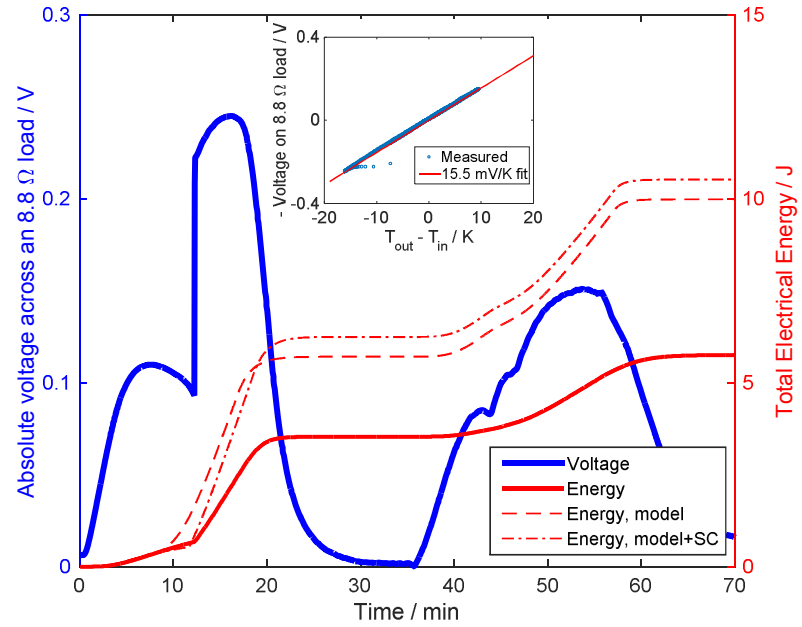

Fig. 6. Voltage and energy output on an $8.8 \Omega$ load of the scaled down prototype, with the Eureca TEG, corresponding to the temperature cycle of Figure 5. The $\mathrm{V}-\Delta \mathrm{T}$ fit is shown in the inset. The modeled energy performance is also plotted for comparison.

Fig. 3 except for the TEG heat resistance which was set to the nominal value given by the Eureca TEG specifications (i.e. $30 \mathrm{~K} / \mathrm{W}$ ), and for the PCM volume which was determined, by fitting of the simulation to the experimental phase change duration, to be $0.9 \pm 0.1 \mathrm{ml}$. The corresponding curves are shown as a dashed and a dash-dot curve in Figure 5 respectively. Better matching in the exponential (non-phase change) regions is obtained, and similar matching in the phase-change gradient. The deviation of phase change temperature is again apparent.

The voltage response, measured across an $8.8 \Omega$ resistive load, and the corresponding cumulative harvested energy are illustrated in Figure 6. The total cumulative energy from a full temperature cycle was $5.5 \mathrm{~J}$, corresponding to an energy density of $3.93 \mathrm{~J}$ per gram of PCM for this implementation. As before, the electrical power and energy output can be simulated, using the simulated $\mathrm{T}_{\text {in }}$ profile and calculating the output voltage through the effective Seebeck coefficient of the system, which can be determined by fitting the measured $\mathrm{V}-\Delta \mathrm{T}$ data as shown in the inset of Figure 6. A value of $15.5 \mathrm{mV} / \mathrm{K}$ is obtained, slightly less than that of the same HSU with the Marlow TEG. As before, both models overestimate the cumulative energy because the transient-induced offset error of the temperature measurements leads to an overestimation of the effective Seebeck coefficient.

\section{Scaled up prototype}

For the scaled up prototype, a $40.1 \times 40.1 \times 3.9 \mathrm{~mm}$ Marlow TG12-6L TEG model was used, with an internal electrical resistance of $3.8 \Omega$ and a thermal resistance of $1.58 \mathrm{~K} / \mathrm{W}$. This corresponds to a thermal conductivity of $1.54 \mathrm{~W} / \mathrm{mK}$. 
Again, the reference to the value of thermal conductivity, in addition to the thermal resistance, for the TEG is given for a model-independent comparison of available technologies.

The heat storage unit comprises an extruded polystyrene lid-less box with outer dimensions $74 \times 135 \times 42 \mathrm{~mm}$ and inner dimensions $48 \times 108 \times 34 \mathrm{~mm}$ and a size matching multi-fin aluminum thermal bridge. The total HSU volume capacity, taking into account the thermal bridge displacement, is $140 \mathrm{~cm}^{3}$. For the experiments, $110 \mathrm{~cm}^{3}$ of water were used as PCM allowing substantial space for expansion during temperature sweep and phase change, which is expected to be at around $10 \%$ of the volume within the range used during experiments. As with the scaled down prototype, the device was characterized in an environmental chamber for various different temperature cycles. The TEG output was connected to a matched $3.8 \Omega$ load.

The temperature response of the scaled-up prototype is presented in Figure 7. The cycle was from $+22{ }^{\circ} \mathrm{C}$ to $-20{ }^{\circ} \mathrm{C}$ and back, with a temperature change rate of around $4 \mathrm{~K} / \mathrm{min}$, similar to the ones presented for the scaled down prototype. The impact of the device heat absorption and release to the performance of the environmental chamber is more pronounced, temporarily disrupting the applied temperature cycle. This effect leads to underestimation of the device performance in a real environment. A $\Delta \mathrm{T}$ of $15{ }^{\circ} \mathrm{C}$ is achieved both during the cooling down and the warming up phases.

In order to simulate the response of the scaled-up prototype, a new set of parameters is required, to reflect the different heat bridge, TEG and amount of PCM used, in comparison with the devices of the previous section. As before, for the HSU thermal resistance R, the nominal value

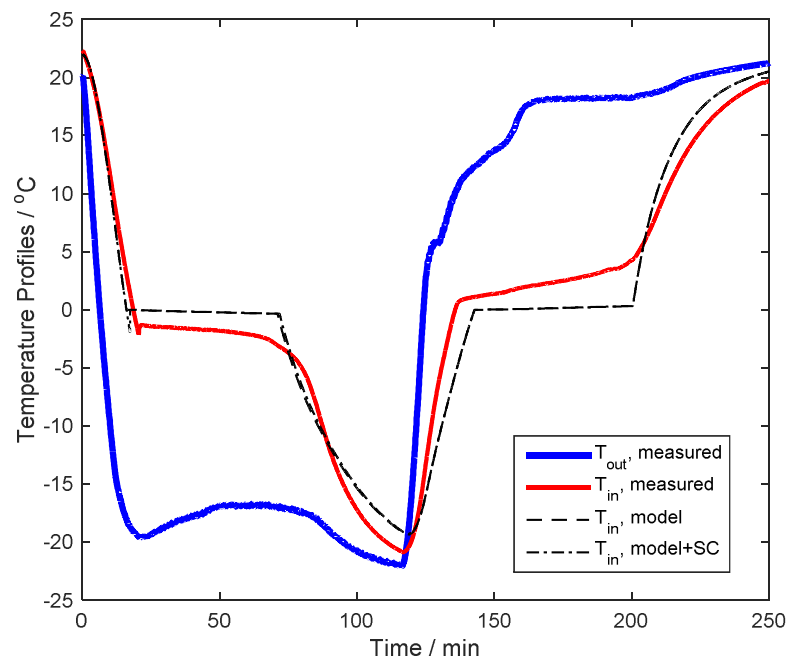

Fig. 7. Temperature response of the scaled-up prototype, with 110 $\mathrm{ml}$ of water as the PCM. Modeled responses are also shown for comparison. The model curves overlap due to weak super-cooling. of the TEG thermal resistance $(1.58 \mathrm{~K} / \mathrm{W})$ was used, neglecting heat leakage through the insulation. The PCM and aluminium masses were $110 \mathrm{~g}$ and $238 \mathrm{~g}$ respectively while the PCM-Al interface surface was $80 \mathrm{~cm}^{2}$. The nominal values for water and aluminium properties were used, taking $0.58 \mathrm{~W} / \mathrm{m} \cdot \mathrm{K}$ as the effective conductivity during phase change. The non-fitted simulation curve matches well to the experimental data, with super-cooling being far less pronounced than in the case of the scaleddown devices. The phase change temperature shift is still present in the experimental data, although also substantially smaller $\left(-1{ }^{\circ} \mathrm{C}\right.$ and $+1{ }^{\circ} \mathrm{C}$ at cool-down and warm-up respectively).

The corresponding voltage output, measured across a resistive load of $3.8 \Omega$, is shown as a bold (blue in online version) curve in Figure 8. Using the same method as with the scaled-down prototypes, an effective Seebeck coefficient of $18.5 \mathrm{mV} / \mathrm{K}$ is found, as shown in the inset. The simulated cumulative energy curves are almost identical to each other and very close to the measured performance of the device. This is due to the very weak super-cooling that is observed (to be discussed in the following section) but also to the slower sweep of $\mathrm{T}_{\mathrm{IN}}$ which occurs due to the significantly larger heat capacitance of the device. This slower temperature change reduces the temperature inhomogeneity in the device and, in turn, the deviation of the measured from the actual $\Delta \mathrm{T}$ on the TEG. Hence, the Seebeck coefficient cumulative energy overestimation is much smaller than that of the smaller devices. The total experimentally measured energy harvested from a full temperature cycle was $254 \mathrm{~J}$. This corresponds to an energy density of $2.31 \mathrm{~J}$ per gram of PCM.

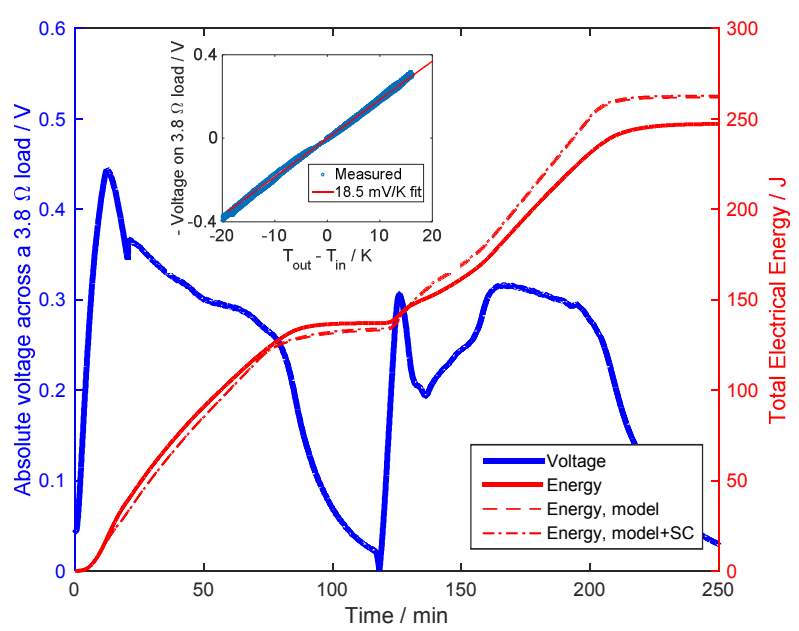

Fig. 8. Voltage and energy output on a $3.8 \Omega$ load of the scaled-up prototype, corresponding to the temperature cycle of Figure 7. The corresponding modeled energy performance is also plotted for comparison, using the effective Seebeck coefficient value determined from the $\mathrm{V}-\Delta \mathrm{T}$ fit shown in the inset. 


\section{Supercooling}

From the performance analysis of the devices presented in this paper, and especially in the smaller devices, it is apparent that super cooling can have a significant effect on the output power of heat storage thermoelectric harvesting devices. Super-cooling causes a reduced $\Delta \mathrm{T}$ during its occurrence. However, the reduced heat flow is preserved for the phase change stage, which usually maintains a higher $\Delta \mathrm{T}$ and hence a more effective time for heat flow and conversion. This is due to the approximately linear dependence of TEG efficiency on the applied $\Delta \mathrm{T}$. Whether super-cooling has a positive or negative effect on the overall output energy depends on the environmental temperature change rate in comparison to the thermal time constant $\mathrm{RC}$ of the device (where $\mathrm{R}$ is the thermal resistance and $\mathrm{C}$ the thermal capacitance of the device). The $\mathrm{RC}$ values of the Marlow and Eureca TEG scaled-down devices and of the scaled-up device are $73 \mathrm{~s}$ and $685 \mathrm{~s}$ for liquid state and 125 $\mathrm{s}$, and $1067 \mathrm{~s}$ for solid state respectively. On the other hand, the $4 \mathrm{~K} /$ minute temperature sweep rate applied in the experiments corresponds to a transition duration of $600 \mathrm{~s}$. It is noted that the relative values of total available sensible and latent heat may also play a significant role in this effect.

The model presented in section 4 was used to simulate the performance of the devices presented in this paper. The super-cooling temperature $\mathrm{T}_{\mathrm{PC}}$ was set directly to the experimentally observed value. The difference in the temperature profiles is apparent in the case of the scaleddown devices (Figures 3 and 5), where super-cooling is pronounced. In terms of device performance, the comparison between simulation results of energy output with and without super-cooling can provide a useful indication. An example can be found in the comparison between the two simulation curves of Figure 4. During the super-cooling stage, the dash-dot (super-cooling included) curve provides less energy to the TEG, as a small $\Delta \mathrm{T}$ is maintained. Hence, by the end of super-cooling (minute 12 of the simulation) the TEG has delivered less energy than in the non - super-cooling case (dashed curve). However, during the phase change stage this is compensated by the release of reserved heat at a higher $\Delta \mathrm{T}$ and hence higher efficiency, leading to an overall energy gain of around $5 \%$. The same gain is observed in Figure 6 for the case of using the Eureca TEG.

As mentioned, no significant super-cooling was observed for the large scale prototype device. This could be attributed to the larger PCM mass, which makes the avoidance of solidification nucleation less likely. In order to draw a reliable conclusion about a correlation between super-cooling and device size, further investigation would be required, taking into account the effects of PCM purity, surface to volume ratio and surface texture but also the possibility of vibration-triggered nucleation.

\section{State of the art of dynamic thermoelectric harvesters}

A summary of the main features and energy density demonstrated by the three scaled devices presented in this paper is given in Table II. For comparison, three other implementations of dynamic thermoelectric harvesting are also included. The performance of such devices depends significantly on the materials used and the design of the heat storage unit, including the thermal bridge and the insulation. Yet, all prototypes yield an energy density between $2.3 \mathrm{~J}$ and $5 \mathrm{~J}$ per gram of PCM. The corresponding maximum theoretically achievable energy density for the particular temperature cycle used and the currently available TEGs (ZT 0.7), is $10 \mathrm{~J}$ per gram of PCM (Kiziroglou et al. 2014).

Table II. Comparison of energy density among different dynamic harvester implementations using water as a PCM.

\begin{tabular}{|c|c|c|c|c|c|}
\hline Organisation/Year & $\begin{array}{c}\text { Total device size } \\
\text { ml }\end{array}$ & TEG & Energy J & $\begin{array}{c}\text { Energy } \\
\text { Density } \\
\mathrm{J} / \mathrm{ml} \\
(\mathrm{PCM}) \\
\end{array}$ & $\begin{array}{l}\text { Energy Density } \\
\mathrm{J} / \mathrm{ml} \text { (device) }\end{array}$ \\
\hline $\begin{array}{l}\text { AIRBUS / } 2008 \\
\text { (Samson et al. 2010) }\end{array}$ & 24 plus Insulation & $\begin{array}{c}4 \times \text { Eureca } \\
\text { TEG1-9.1-9.9-0.8/200 }\end{array}$ & 35 & 3.5 & $\begin{array}{l}1.5 \text { (without } \\
\text { insulation) }\end{array}$ \\
\hline $\begin{array}{l}\text { LAAS-CNRS / } 2008 \text { (Bailly et al. } \\
\text { 2008) }\end{array}$ & - & Micropelt MPGD602 & 34 & 2.8 & - \\
\hline Imperial / 2014 (Kiziroglou et al. 2014) & 78 & 2 x Marlow TG12-2-5 & 105 & 4.57 & 1.3 \\
\hline $\begin{array}{l}\text { Imperial and AIRBUS/2015 } \\
\text { (This paper, scaled-down 1) }\end{array}$ & 9.7 & Marlow NL1013T & 5.8 & 4.14 & 0.6 \\
\hline $\begin{array}{l}\text { Imperial and AIRBUS/2015 } \\
\text { (This paper, scaled-down 2) }\end{array}$ & 9.7 & $\begin{array}{c}\text { Eureca } \\
\text { TEG1-9.1-9.9-0.8/200 }\end{array}$ & 5.5 & 3.93 & 0.6 \\
\hline $\begin{array}{l}\text { Imperial and AIRBUS/2015 } \\
\text { (This paper, scaled-up) }\end{array}$ & 420 & Marlow TG12-6L & 254 & 2.31 & 0.6 \\
\hline
\end{tabular}

This work has been published as: Scaling and super-cooling in heat storage harvesting devices, Micro System Technologies, 22, 1905-1914, DOI 10.1007/s00542-016-2889-0, 2016. 


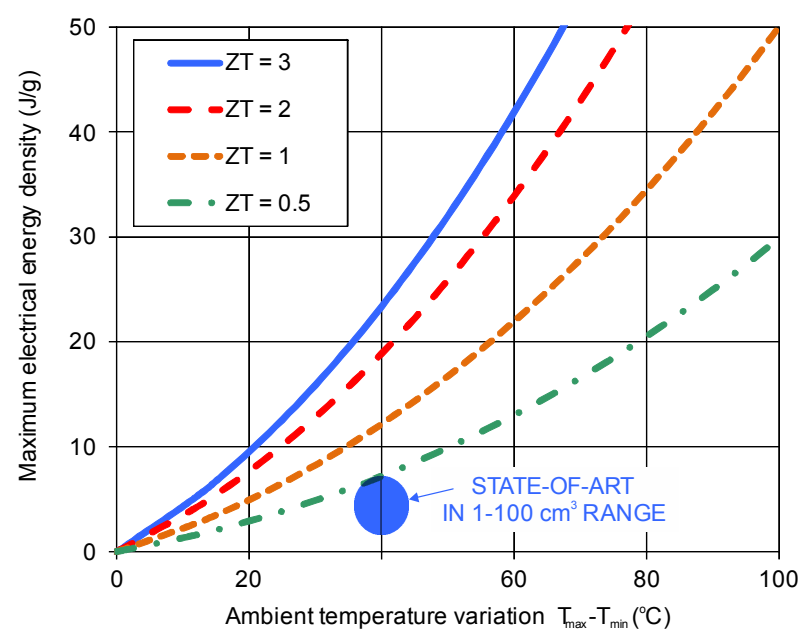

Fig. 9. State-of-the-art of performance for dynamic thermoelectric harvesting prototypes in comparison to the theoretical maximum electrical energy density for different TEG technologies.

The state-of-the-art of performance for dynamic thermoelectric harvesting prototypes is illustrated in Figure 9, in comparison to the theoretical maximum electrical energy density for different TEG technologies.

The demonstrated electrical energy for all prototypes reported to date is plotted against PCM volume in Figure 10. No increasing or decreasing trend can be identified for the energy density as a function of size. Nevertheless, smaller (or flatter) HSU designs in general may allow a smaller heat path and decreased temperature inhomogeneity in the PCM, meaning lower $\Delta \mathrm{T}$ loss and higher conversion efficiency. It can be concluded that devices in the volume range $1-100 \mathrm{~cm}^{3}$ can provide a useful amount of energy for their size. An instructive indication of power availability can be found in the comparison of performance, in terms of $\mathrm{J}$ per PCM volume, against state of the art non-rechargeable batteries. The $2.3-4.6 \mathrm{~J} / \mathrm{g}$ output of the dynamic thermoelectric harvesting prototypes will reach the energy density of an alkaline battery $(1.8 \mathrm{~kJ} / \mathrm{ml}$ (Energizer 2016)) after 400-800 cycles of operation and a lithium one (2.4 $\mathrm{kJ} / \mathrm{ml}$ (Panasonic 2016)) after 500-1000 cycles. Performance degradation is not expected due to the solid state nature of the TEGs and the chemically passive nature of the phase change materials used, contrary to chemical energy storage media like batteries. With regard to the observation and discussion of the super-cooling effect, it is expected that applications with high temperature sweep rates would benefit from suppression of super cooling. In contrast, applications with low temperature sweep rates could benefit from (or, under certain conditions, even rely on) super-cooling. In order to distinguish between the two cases, the thermal time constant RC, the PCM volume and the size of the device should be taken into account.

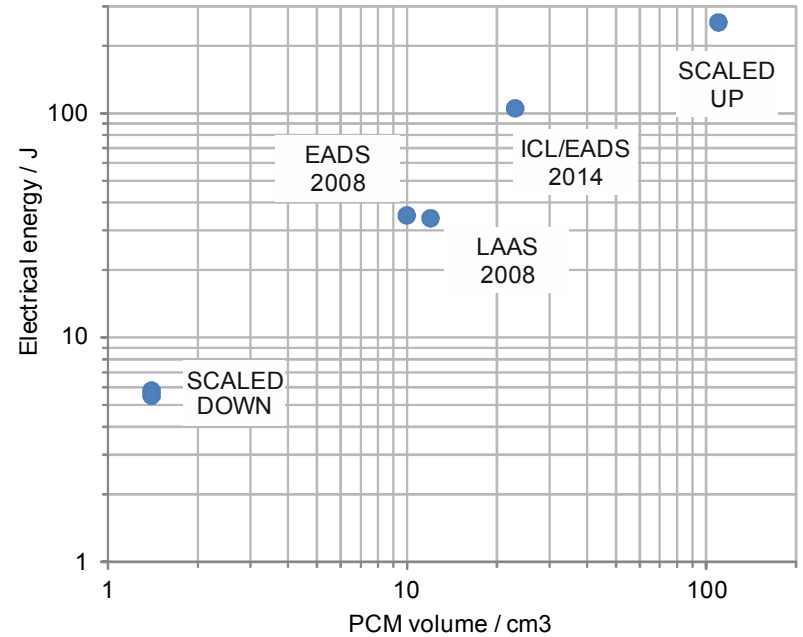

Fig. 10. Demonstrated electrical energy of recent dynamic thermoelectric harvesters as a function of PCM volume.

Particularly, the indication that super-cooling in smaller devices could be both more likely and favourable is important for applications with small device sizes and slow temperature fluctuations.

Further studies on the scaling of dynamic thermoelectric harvesting devices could involve a combined multiparameter analysis and numerical simulation. This could identify new methods and device designs, optimized for particular use cases.

\section{References}

Bailly N, Dilhac J-M, Escriba C, Vanhecke C, Mauran N, Bafleur $\mathrm{M}$ Energy scavenging based on transient thermal gradients: Applications to structural health monitoring of aircraft. In: PowerMEMS, 2008. pp 205-208

Becker T, Elefsiniotis A, Kiziroglou ME (2014) Thermoelectric Energy Harvesting in Aircraft. In: Briand D, Roundy S, Yeatman E (eds) Micro Energy Harvesting. Wiley, pp 415-433

Briand D, Yeatman E, Roundy S (2015) Micro Energy Harvesting. doi:10.1002/9783527672943

Elefsiniotis A, Samson D, Becker T, Schmid U (2013) Investigation of the Performance of Thermoelectric Energy Harvesters Under Real Flight Conditions Journal of Electronic Materials 42:2301-2305 doi:10.1007/s11664-012-2411-0

Energizer (2016) EN91 AA alkaline battery datasheet.

Glynne-Jones P, Tudor MJ, Beeby SP, White NM (2004) An electromagnetic, vibration-powered generator for intelligent sensor systems Sensors and Actuators A: Physical 110:344349

Kiziroglou M, Elefsiniotis A, Wright S, Toh T, Mitcheson P, Becker T, Yeatman E (2013) Performance of phase change materials for heat storage thermoelectric harvesting Applied Physics Letters 103:193902 
Kiziroglou ME, Wright SW, Toh TT, Mitcheson PD, Becker T, Yeatman EM (2014) Design and Fabrication of Heat Storage Thermoelectric Harvesting Devices Industrial Electronics, IEEE Transactions on 61:302-309 doi:10.1109/tie.2013.2257140

Mitcheson PD, Green TC, Yeatman EM, Holmes AS (2004) Architectures for vibration-driven micropower generators Microelectromechanical Systems, Journal of 13:429-440

Panasonic (2016) Coin type lithium batteries (CR series). Panasonic. http://industrial.panasonic.com/ww/products/batteries/primar $\mathrm{y}$-batteries/lithium-batteries/coin-type-lithium-batteries-crseries. Accessed 09.02.2016 2016

Papachristou AA, Kiziroglou ME, Petrou L, Koundouras S, Hatzopoulos AA (2013) Viability of Thermoelectric Energy Harvesting for Precision Agriculture Sensor Nodes.
Paper presented at the 8th Jordanian International Electrical and Electronics Engineering Conference, Amman, Jordan, 1618 Apr.

Samson D, Otterpohl T, Kluge M, Schmid U, Becker T (2010) Aircraft-Specific Thermoelectric Generator Module Journal of Electronic Materials 39:2092-2095 doi:10.1007/s11664-0090997-7

Shenck NS, Paradiso JA (2001) Energy scavenging with shoemounted piezoelectrics Ieee Micro 21:30-42

Sodano HA, Inman DJ, Park G (2005) Comparison of piezoelectric energy harvesting devices for recharging batteries Journal of Intelligent Material Systems and Structures 16:799-807 doi:10.1177/1045389x05056681

Toh TT, Wright SW, Kiziroglou ME, Mitcheson PD, Yeatman EM (2014) A dual polarity, cold-starting interface circuit for heat storage energy harvesters Sensors and Actuators A: Physical 211:38-44 doi:http://dx.doi.org/10.1016/j.sna.2014.02.037 